\title{
Mosaicplasty by a Modified Lateral Malleolus Osteotomy Approach for Osteochondral Lesion of Talus
}

\author{
BS Rajsirish ${ }^{1}$, Schiller Jos ${ }^{2}$ \\ ${ }^{1}$ Department of Orthopaedics, Muller Ortho Hospital, Chennai, Tamil Nadu, India; ${ }^{2}$ Department of Arthoscopy and Sports Medicine, \\ Baby Memorial Hospital, Kozhikode, Kerala, India.
}

\section{Corresponding Author: \\ Dr. Rajsirish BS \\ Email: dr.rajsirish@gmail.com}

This is an Open Access article distributed under the terms of the Creative Commons Attribution License (creativecommons.org/ licenses/by/3.0).

Received : May 15,2020

Accepted : July 21, 2020

Published : August 30, 2020

\begin{abstract}
Background: Osteochondral lesions of talus commonly affect the medial and anterolateral dome. These acquired or idiopathic lesions of the articular cartilage are least likely to affect the posterolateral part of the dome. Furthermore, if encountered, they are difficult to manage due to the limited surgical exposure. Case Report: We report a case of idiopathic osteochondral lesion of the lateral talar dome in a 40-year-old male. He presented with recurrent ankle effusion and pain, disabling his activities of daily living. He was managed by osteochondral autologous grafting by a modified lateral malleolus osteotomy approach. Osteotomy site was fixed with $1 / 3^{\text {rd }}$ tubular plate. Patient improved clinically. Conclusion: To overcome the complications associated with the traditional lateral transmalleolar approach we made certain modifications like keeping the anterior talofibular ligament and calcaneofibular ligament intact. We believe this helps in minimizing lateral instability and could encourage more surgeons to take up this approach.
\end{abstract}

Keywords: Ankle Joint, Fibula, Lateral Ligament, Osteotomy, Pain.

\section{Introduction}

Osteochondral lesions are acquired or idiopathic lesions of subchondral bone than can produce delamination and sequestration with the articular cartilage involvement and instability [1]. It is estimated that up to $50 \%$ of acute ankle sprains and fractures will develop some form of osteochondral injury [2]. It is most frequently seen in the second decade of life but can persist later [3]. Classically in talus they are divided into anterolateral and posteromedial lesions of the dome. With the invent of cartilage sensitive imaging modalities their incidence is increasing, with more and more reports of unusual patterns also detected. Among the different locations, lesions of the lateral talar dome are difficult to treat probably due to their lower incidence of spontaneous healing and constraints in surgical exposure. For medial talar dome, most often surgeons revert to a medial malleolus or distal tibia osteotomies for adequate exposure. But for lateral talar dome, due to their rarity, such methods for improving their surgical exposure are not often discussed in detail.

\section{Case Report}

A 40-year-old male presented with 4 months history of recurrent pain and swelling of the left ankle. Pain aggravated on movement and was impeding his activities of daily living. He had no history of trauma or any relevant medical/personal history. Clinically there was effusion in the ankle joint with diffuse tenderness. Range of motion was restricted terminally. He had no features of ankle instability. Initial blood investigations and $\mathrm{X}$-rays were unremarkable. Magnetic resonance imaging revealed a $16 \mathrm{~mm}$ grade III (Hepple et al.) osteochondral defect in the lateral talar dome posteriorly with a degenerative subchondral 
cyst and marked oedema of the adjacent marrow [Fig.1]. As the lesion was not detached a trial of conservative management was given with below knee cast and non-weight bearing. After two months of conservative management, the patient continued to have similar complaints. Hence it was decided to go for operative management.

Our pre-operative planning included mapping of the lesion, deciding the approach and whether to do a mega OATS or mosaicplasty. On mapping we found the lesion was located more posteriorly on the lateral talar dome. It would be impossible to obtain adequate exposure by the conventional lateral approach, medial transmalleolar malleolus or distal trans-tibial osteotomy approaches. Few surgeons have reported a lateral trans-malleolus approach for such difficult locations. But they were complicated by nonunion and chronic lateral instability. We decided to modify the classic Campbell approach in case necessary, to overcome these complications by making an oblique osteotomy to minimize nonunion and approaching the talus without disturbing the important lateral ligamentous complex. The lesion was $16 \mathrm{~mm}$, we decided to do mosaicplasty for the obvious reasons of graft integration and donor site morbidity.

The patient was positioned supine and surgery proceeded under spinal anaesthesia and tourniquet control. We preceded by the conventional anterolateral (sagittal) approach along the lateral side of extensor digitorum longus in front of lateral malleolus [Fig.2]. The exposure was inadequate; hence we extended the approach through lateral malleolar osteotomy. The proximal incision was extended to $4 \mathrm{~cm}$ above the tip of the lateral malleolus. After exposing the distal fibula anterolaterally, an oblique osteotomy was performed (after predrilling the holes for final fixation) $1 \mathrm{~cm}$ above the level of the syndesmosis. Both the anterior and posterior syndesmotic ligaments were cut and tagged. All the other ligaments anterior talofibular, calcaneofibular and posterior talofibular ligaments were left intact, with these ligaments as fulcrum, lateral malleolus was flipped [Fig.3]. With ankle in plantarflexion, varus and inversion the entire lateral talar dome
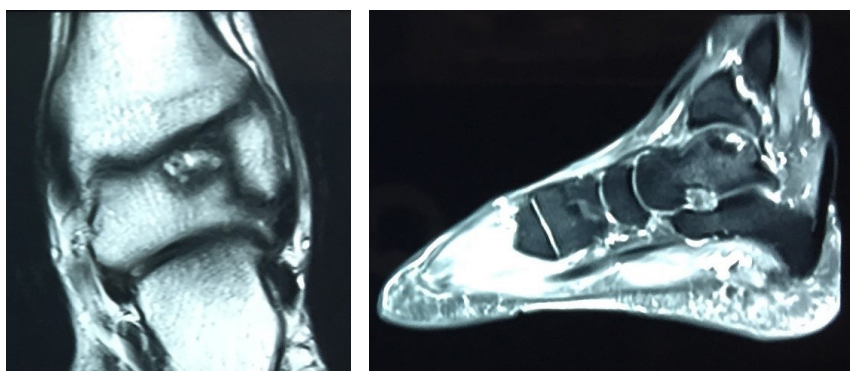

Fig.1: MRI of the ankle joint showing an osteochondral defect in the posterolateral aspect of the superior articular surface of talus and a corresponding subchondral cyst.

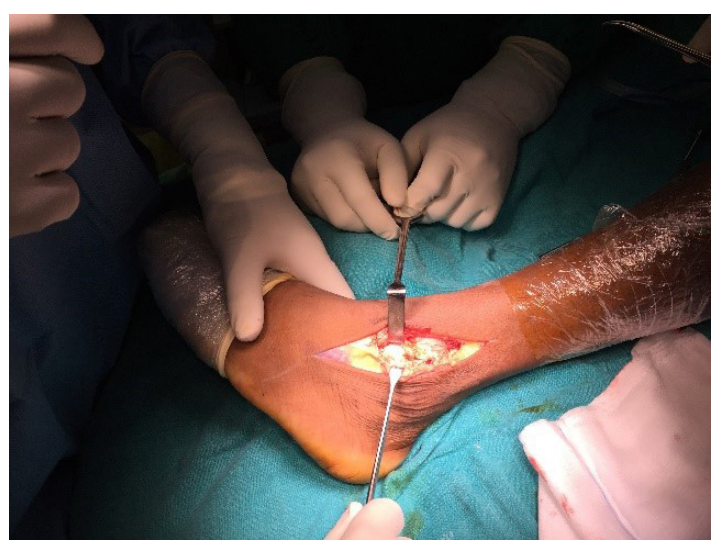

Fig.2: The classical anterolateral (sagittal) approach as described by Campbell. Arthrotomy done to examine the lesion is inadequate for the procedure.

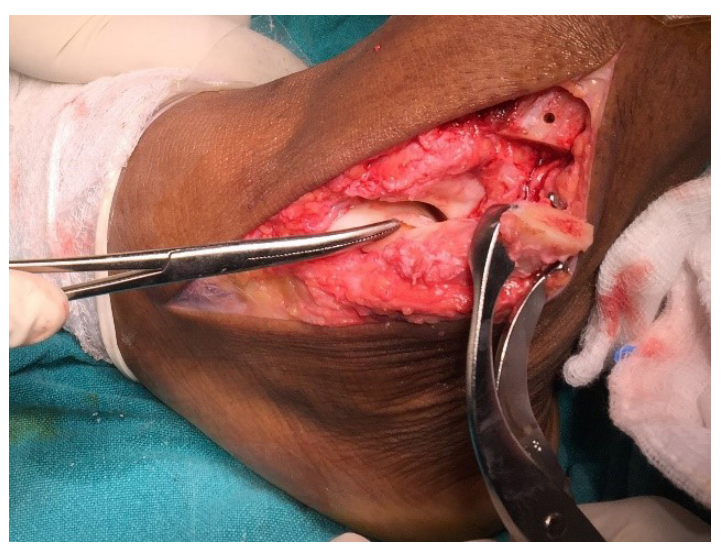

Fig.3: Completed oblique osteotomy of lateral malleolus with predrilled screw holes. Undetached osteochondral lesion and the underlying soft bone visualized. 
could be visualized. As talus is approached through the osteotomy from above, manoeuvring the long instruments for mosaicplasty and placement of graft perpendicular to the defect would become hassle free.

The detached osteochondral fragment (Pritsch Stage III) was debrided and the defect was freshened using a curette. A huge subchondral cyst was present. Cyst wall was debrided to include it in the defect and prepared for receiving the graft. Base of the defect was drilled with $2 \mathrm{~mm}$ smooth $\mathrm{K}$-wires to increase the vascularity [Fig.4]. We used Arthrex Osteochondral Autologous Transport System-Apple Core technique [Fig.5]. The total defect after preparation was measured to be 18 $\mathrm{mm}$. Three $6 \mathrm{~mm}$ osteochondral cylindrical grafts

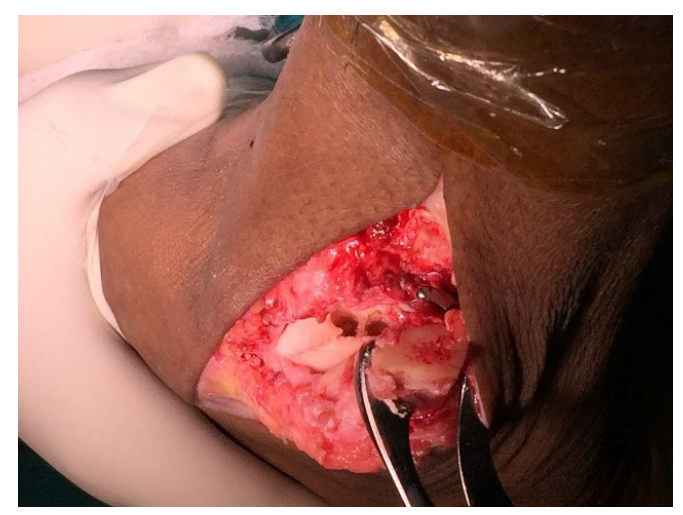

Fig.4: Serial preparation of the defect by curetting and drilling the base of the lesion to increase vascularity. Three $6 \times 8 \mathrm{~mm}$ cores were made to accommodate the graft.

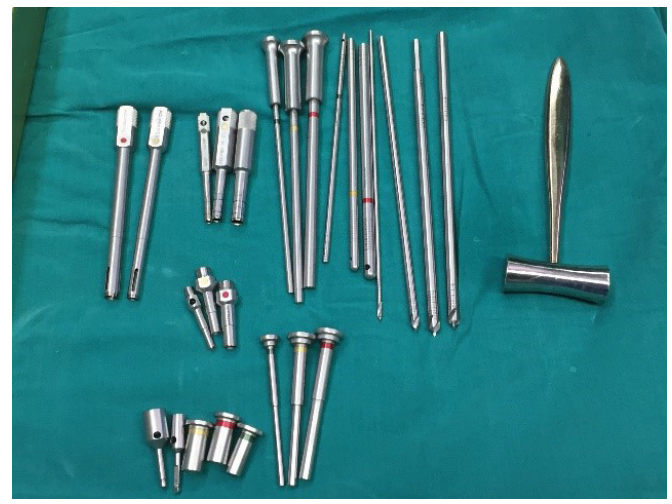

Fig.5: Arthrex instrumentation for graft harvesting and recipient site preparation. of $13 \mathrm{~mm}$ depth were harvested from ipsilateral non-weight bearing part of lateral femoral condyle [Fig.6]. Grafts were plugged into the defect without any gap or articular step [Fig.7]. Direct repair of ankle joint capsule, anterior and posterior syndesmotic ligaments were performed. Lateral malleolus was fixed with $1 / 3^{\text {rd }}$ tubular plate and screws [Fig.8]. Post-fixation ankle joint was stable, so syndesmotic fixation was deferred. With stability provided by the interosseous membrane and lateral ligaments the need for syndesmotic fixation is controversial. Ankle was immobilized in a below knew slab.

Post-operatively the patient was started on non-weight bearing mobilization from day one. X-ray showed good reduction and fixation of lateral

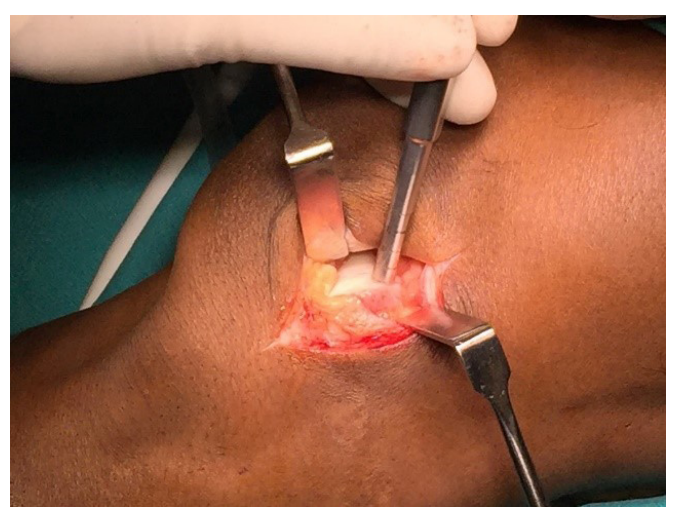

Fig.6: Three $6 \mathrm{~mm}$ diameter and $8 \mathrm{~mm}$ depth osteochondral grafts harvested from the ipsilateral non-articular part of lateral femoral condyle.

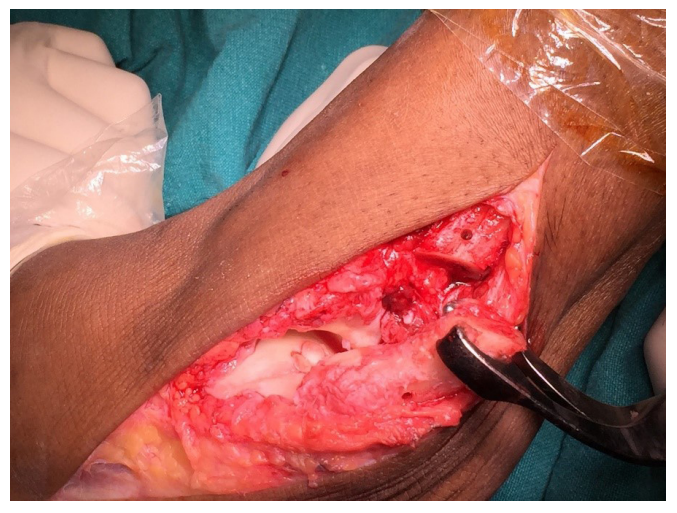

Fig.7: Graft placement in the prepared defect. 
malleolus and stable ankle joint. Serial wound inspections were done and sutures were removed on the $14^{\text {th }}$ day. After suture removal below knee cast was applied and continued the same for 2 months. He started partial weight bearing over cast at 2 months. At the end of three months, cast was removed. Ankle ROM exercises were started. Graduated weight bearing for 2 weeks followed by strengthening, balance training and proprioception exercises were given. The above was continued till 6 months post procedure.

Serial radiographs showed union at the osteotomy site [Fig.9]. At the end of 6 months patient has full range of motion at ankle and

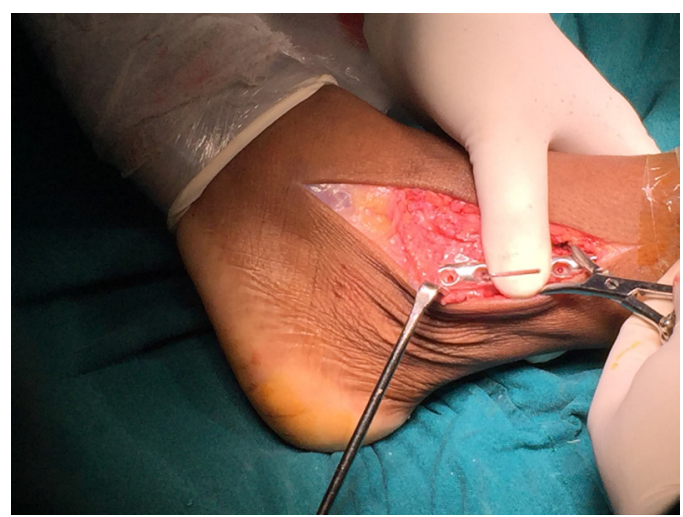

Fig.8: Image showing the surgical site after capsule and syndesmotic ligaments repair. Lateral malleolus was fixed with $1 / 3^{\text {rd }}$ tubular plate and screws.

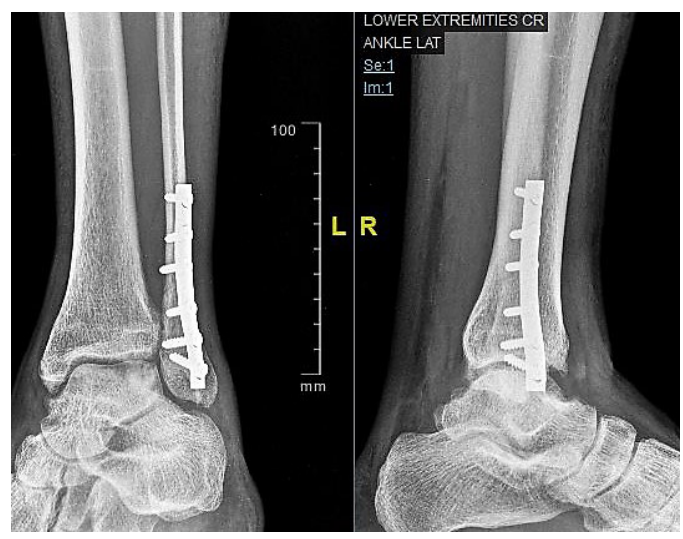

Fig.9: Post-operative radiograph showing well reduced syndesmosis and union at the osteotomy site. a stable joint. He had asymptomatic and pain-free mobilization. MRI taken at the end of 8 months showed a stable graft with integration, no effusion, no articular step and uniform articular cartilage [Fig.10]. In Figure 11 completely healed syndesmotic ligaments can be seen. The modified MOCART scoring system based on the MRI was 75/85 at 8 months [Table 1].

\section{Discussion}

Osteochondral lesions of talus are uncommon but can lead to pain, disability and affect

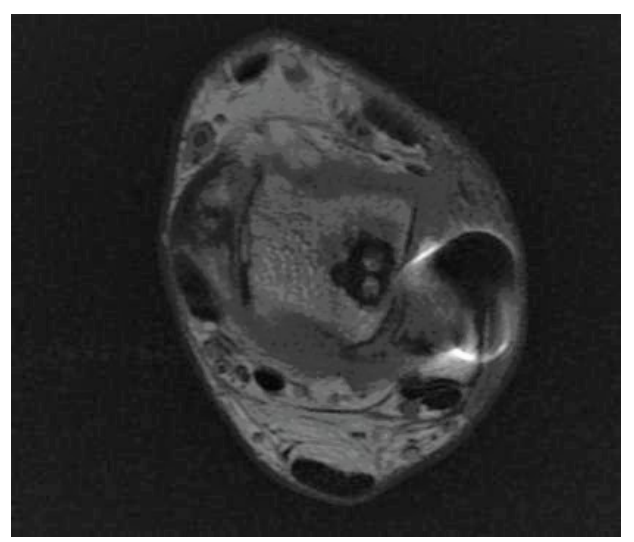

Fig.10: Post-operative MRI at the end of one year showing complete filling of the defect and graft take up.

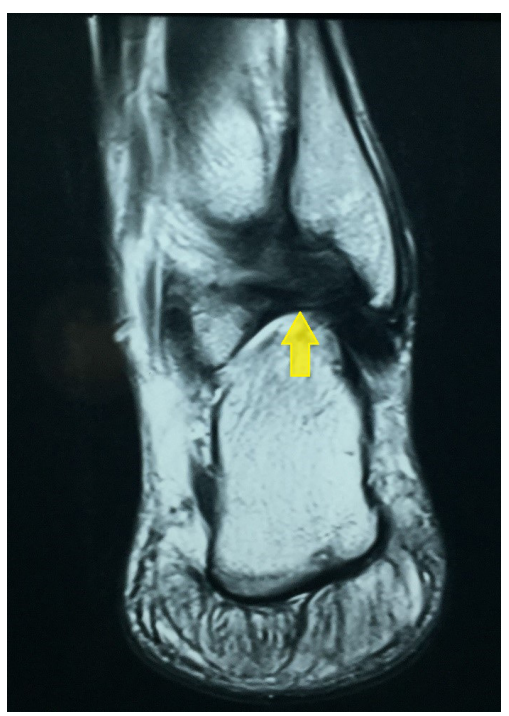

Fig.11: Arrow showing healed posterior syndesmotic ligament. 
activities of daily living [4]. In recent years various biomechanical studies have provided us with a better understanding of the pathology. Subsequently various classification systems have been proposed based on the characteristics in X-ray (Berndt \& Harty's) [5], CT and MRI. MRI based Hepple [6] and arthroscopy based Pritsch staging are more commonly used. In asymptomatic patients' non-operative management like rest, restriction of activities and NSAIDs can be tried. Zengerink et al. reported a $45 \%$ success rate with conservative management [7]. But more than half of these patients have progressive worsening.

Over the years various forms of operative treatments have been used like bone marrow stimulation, radiofrequency ablation, microfracture, abrasion chondroplasty, osteochondral autologous transfer, mega OATS, autologous chondrocyte implantation- collage and matrix induced [8]. Though various techniques are available, the advantage of replacing like with like is biomechanically superior [9]. In a systematic review Zengerink et al. demonstrated $87 \%$ results in various studies with OATS or mosaicplasty. The main concern is the donor site morbidity. But properly selected donor site and graft harvested from non-weight bearing area have showed minimal morbidity [9]. Though recent trend is towards MACI and CACI, mosaicplasty still is a reasonable procedure for defects of size less than $4 \mathrm{~cm}$.

Difference in depth or shape of graft as it sits in talus will dictate the difference between success and failure [10]. Nam et al. showed that these grafts in time will remodel and mimic the surrounding cartilage [11]. Hangody et al. demonstrated good to excellent results in 24/26 cases treated with open OATS under 7 years follow up. We followed a similar technique as described by Hangody et al. with multiple grafts nested together [12]. The lateral transmalleolar approach, is an oblique osteotomy of the lateral malleolus just above the syndesmosis. Anterior syndesmotic and anterior
Table 1: Modified MOCART score on MRI.

\begin{tabular}{|l|l|l|}
\hline Variable Assessed & $\begin{array}{l}\text { MR } \\
\text { Characteristics }\end{array}$ & $\begin{array}{l}\text { Point scale as per } \\
\text { MOCART }\end{array}$ \\
\hline $\begin{array}{l}\text { Degree of defect } \\
\text { filling }\end{array}$ & Near complete & $20 / 20$ \\
\hline $\begin{array}{l}\text { Integration with } \\
\text { border zone }\end{array}$ & Complete & $15 / 15$ \\
\hline $\begin{array}{l}\text { Quality of repair } \\
\text { tissue surface }\end{array}$ & Smooth & $10 / 10$ \\
\hline $\begin{array}{l}\text { Structure of repair } \\
\text { tissue }\end{array}$ & Inhomogeneous & $0 / 10$ \\
\hline $\begin{array}{l}\text { T2 signal intensity } \\
\text { of repair tissue }\end{array}$ & $\begin{array}{l}\text { Moderately } \\
\text { hyperintense }\end{array}$ & $5 / 15$ \\
\hline Subchondral lamina & Intact & $5 / 5$ \\
\hline $\begin{array}{l}\text { Subchondral bone } \\
\text { sclerosis }\end{array}$ & No & $5 / 5$ \\
\hline Joint effusion & Absent & $5 / 5$ \\
\hline
\end{tabular}

talofibular ligaments are cut. If further exposure is needed calcaneofibular ligament is also cut [9]. The lateral malleolus is then displaced posteriorly and inferiorly with posterior syndesmotic ligaments and posterior talofibular ligaments as a hinge. There is limited data on this approach. Even those studies have reported complications of non-union, lateral instability and syndesmotic widening.

To overcome these complications, we modified the approach. Talus was exposed by keeping the anterior talofibular ligament and calcaneofibular ligament intact, thus minimizing the complications of lateral instability. The anterior and posterior syndesmotic ligaments were cut and tagged. Lateral malleolus is flipped distally just enough for visualizing lateral talar dome and maneuvering long arthroscopic instruments. Lateral malleolus is not stripped of the soft tissues for exposure. The nonunion can be prevented by creating osteotomy at the metaphyseal region with increased obliquity. Direct repair of the syndesmotic ligaments or by pull through technique minimize the syndesmotic instability. It can be further augmented with syndesmotic screws if needed. Our case had normal union without any instability at the end of one year. Apart from the above advantages, we could get an easier end on visualization of the 
lateral talar dome and maneuvering of the long instruments for the perfect graft placement was also better.

\section{Conclusion}

Though a large cohort is needed to confirm these findings, we hypothesize that these modifications can help limit the complications and encourage more surgeons to take up this approach.

Contributors: BSR: manuscript editing, patient management and the conception of the work; SJ: manuscript editing, literature review and patient management; BSR will act as a study guarantor. Both authors approved the final version of this manuscript and are responsible for all aspects of this study.

Funding: None; Competing interests: None stated.

\section{References}

1. Zanon G, DI Vico G, Marullo M. Osteochondritis dissecans of the talus. Joints. 2014;2:115-123.

2. Saxena A, Eakin C. Articular talar injuries in athletes: Results of microfracture and autogenous bone graft. Am J Sports Med. 2007;35:1680-1687.

3. Bruns J, Rosenbach B. Osteochondrosis dissecans of the talus. Arch Orthop Trauma Surg. 1992;112:23.

4. Gianni S, Buda R, Vannini F, Caprio FD, Grigolo B. Arthroscopic autologous chrondrocyte implantation in osteochondral lesions of the talus: surgical technique and results. Am J Sports Med. 2008;36:873-880.

5. Berndt AL, Harty $M$. Transchondral fractures (osteochondritis dissecans) of the talus. J Bone Joint Surg (Am). 1959;4:1363-1363.

6. Hepple S, Winson IG, Glew D. Osteochondral lesions of the talus: a revised classification. Foot Ankle Int. 1999; 20:789-793.

7. Zengerink M, Struijs PA, Tol JL, van Dijk CN. Treatment of osteochondral lesions of the talus: a systematic review. Knee Surg Sports Traumatol Arthrosc. 2010;18:238-246.

8. Arianna LG, Youichi Y, Charles PH, John GK. Current management of talar osteochondral lesions. World Journal of Orthopedics. 2017;8:12-20.

9. John GK, Christopher DM. The treatment of osteochondral lesions of the talus with autologous osteochondral transplantation and bone marrow aspirate concentrate: Surgical technique. Cartilage. 2011;2:327336.

10. Huang FS, Simonian PT, Norman AG, Clark JM. Effects of small incongruities in a sheep model of osteochondral autografting. Am J Sports Med. 2004;32:1842-1848.

11. Nam EK, Makhsous M, Koh J. The effect of graft height mismatch on contact pressure following osteochondral grafting: a biomechanical study. Am J Sports Med. 2004;32:317-320.

12. Hangody L, Kish G, Modis L. Mosaicplasty for the treatment of osteochondritis dissecans of the talus: two to seven-year results in 36 patients. Foot Ankle Int. 2001;22:552-558. 